\title{
Atopic dermatitis-like skin lesions are suppressed in fat-1 transgenic mice through the inhibition of inflammasomes
}

\author{
Hyun-Young Jang ${ }^{1}$, Jeung-Hyun Koo ${ }^{1}$, Sang-Myeong Lee ${ }^{2}$ and Byung-Hyun Park ${ }^{1}$
}

\begin{abstract}
Previous clinical trials have addressed the beneficial effects of fish oil supplementation on atopic dermatitis. Recently, we reported that fat -1 mice, which can convert $n-6$ to $n-3$ polyunsaturated fatty acids (PUFAs), are protected against allergic airway inflammation because their Th2 immune responses are suppressed. Here, we examined the effects of endogenously synthesized n-3 PUFAs on atopic dermatitis, a representative Th2-dominant allergic inflammatory disease. Mouse models of atopic dermatitis-like skin lesions were prepared by epicutaneous application of 2,4dinitrochlorobenzene (DNCB) or house dust mite (HDM) extract to the ears. DNCB-treated fat-1 mice exhibited markedly reduced epidermal thickening, lower mast cell infiltration, and lower serum lgE and histamine compared with wild-type mice. The draining lymph nodes of fat-1 mice were substantially smaller and contained significantly smaller proportions of activated CD4 ${ }^{+}$T cells and IL-4-producing Th2 cells than those of wild-type mice. Consistent with these findings, the mRNA levels of Th2 cytokines were significantly decreased in DNCB-sensitized skin lesions of fat-1 mice. Lastly, inflammasome activation, IL-1 $\beta$ production, and pyroptotic cell injury were suppressed in fat-1 mice. Similar results were observed in HDM-challenged fat- 1 mice. This study confirms the results of previous clinical studies and suggests fish oil supplementation as a therapeutic strategy for atopic dermatitis-like skin lesions.
\end{abstract}

\section{Introduction}

Atopic dermatitis is a multifactorial chronic inflammatory disease that results from complex interactions between genetic and environmental factors ${ }^{1}$. Atopic dermatitis is also considered a T-helper 2 (Th2)-mediated allergic disease because interleukin (IL)-4, IL-5, and IL-13 produced by Th2 cells play key roles in its onset and development ${ }^{2-4}$. These cytokines stimulate B cells, mast cells, and epidermal cells, leading to IgE production and mast cell degranulation as well as the production of various cytokines. Thymic stromal lymphopoietin (TSLP), a cytokine produced mainly by epidermal keratinocytes and mast cells, promotes the differentiation of naïve $\mathrm{T}$ cells

\footnotetext{
Correspondence: Byung-Hyun Park (bhpark@jbnu.ac.kr)

${ }^{1}$ Department of Biochemistry, Chonbuk National University Medical School,

Jeonju, Jeonbuk 54896, Republic of Korea

${ }^{2}$ Division of Biotechnology, College of Environmental and Bioresource

Sciences, Chonbuk National University, Iksan, Jeonbuk 54596, Republic of Korea

The authors contributed equally: Hyun-Young Jang, Jeung-Hyun Koo.
}

into Th2 cells ${ }^{5}$. TSLP is highly expressed in skin lesions of atopic dermatitis patients ${ }^{6,7}$. Interestingly, $\mathrm{T}$ cell polarization in atopic dermatitis is biphasic: there is a predominantly a Th2 response in the acute phase, while high levels of interferon (IFN) $-\gamma$ and the appearance of Th1 cells have been observed in the chronic phase ${ }^{8}$. In addition to adaptive immune responses, the innate immune system is activated via evolutionarily conserved pathogen-recognition receptors, such as Toll-like receptors (TLRs) and nucleotide-binding oligomerization domain receptors (NLRs) ${ }^{6,9}$. The most well known of these is NLR family pyrin domain-containing protein 3 (NLRP3), which recruits apoptosis-associated speck-like protein containing a caspase recruitment domain (ASC) and pro-caspase-1. Inflammasome assembly then activates caspase- 1 and leads to the release of IL- $1 \beta$ and IL$18^{10}$. These ILs are important in innate immune functions and in the regulation of adaptive immune responses. 
Indeed, single-nucleotide polymorphisms (SNPs) in the Nlrp3 gene are associated with atopic dermatitis ${ }^{11-13}$.

Based on the pathology of atopic dermatitis, immunomodulating strategies have been developed to treat the disease. These strategies include corticosteroids, calcineurin inhibitors, and IgE-neutralizing antibodies ${ }^{14-16}$. However, prolonged use of these drugs often produces adverse effects in atopic dermatitis patients. Therefore, a safe and long-term therapeutic strategy is required. Several epidemiological studies have shown that dietary fish oil supplementation decreases the prevalence of atopic dermatitis and alleviates the severity of the disease ${ }^{17}$. Fish oil is an important source of $n-3$ polyunsaturated fatty acids (PUFAs), such as eicosapentaenoic acid (EPA, 20:5) and docosahexaenoic acid (DHA, C22:6). The incorporation of $n-3$ PUFAs into the membranes of immune cells, at the expense of arachidonic acid, is thought to be important for the anti-inflammatory effects of $n-3$ PUFAs ${ }^{18}$, which gives rise to fewer inflammatory eicosanoids. This shift ultimately affects cytokine production. n3 PUFAs suppress T-cell activation ${ }^{19}$, monocyte chemotaxis $^{20}$, macrophage M1 polarization ${ }^{21}$, and dendritic cell maturation ${ }^{22}$, resulting in a decrease in local tissue inflammation. However, it is difficult to ascertain the contribution of n-3 PUFAs per se without the potential confounding effects of other dietary components, such as the nutrient composition, total caloric intake, duration of feeding, and contamination of trace elements. To address this issue, Kang et al. $^{23}$. developed fat-1 mice, which contain the fat-1 gene from Caenorhabditis elegans and are able to convert n-6 to n-3 PUFAs in vivo. As a result of the endogenously synthesized n-3 PUFAs in blood and tissues, these mice exhibit the beneficial effects of $n-3$ PUFAs in various inflammatory disease models, such as pancreatitis $^{24}$, allergic airway inflammation ${ }^{19}$, and rheumatoid arthritis ${ }^{25}$. In this study, we investigated whether atopic dermatitis-like symptoms and skin lesions observed in 2,4-dinitrochlorobenzene (DNCB)- or house dust mite (HDM) extract-exposed mice would be alleviated in fat-1 mice. Special attention was paid to the effects exerted by endogenously synthesized n-3 PUFAs on inflammasome activation in skin tissues.

\section{Materials and methods \\ Animals}

Dr. JX Kang of Harvard Medical School (Boston, MA, USA) kindly provided the fat- 1 transgenic mice. Mice were housed in a laminar flow cabinet and maintained on an AIN-76A diet containing 5\% corn oil provided ad libitum. All animal experiments were performed in accordance with the Guide for the Care and Use of Laboratory Animals, published by the US National Institutes of Health (NIH Publication No. 85-23, revised 2011). The current study protocol was also approved by the
Institutional Animal Care and Use Committee of Chonbuk National University (Permit No. CBNU 2017-0085).

\section{Induction of experimental atopic dermatitis}

To induce experimental atopic dermatitis-like skin lesions, the surfaces of both ears were very gently stripped five times with surgical tape (Tegaderm; $3 \mathrm{M}$, St Paul, MN, USA). Mice were sensitized by applying $20 \mu \mathrm{l}$ of $1 \%$ DNCB (Sigma-Aldrich, St. Louis, MO, USA) or vehicle alone to the ear on Day -7. On Day 0, mice were challenged again by applying $20 \mu \mathrm{l}$ of $0.5 \% \mathrm{DNCB}$ to the ears every other day for up to 42 days. Changes in ear thickness were measured weekly. The mice were sacrificed on Day 43. In the HDM (Dermatophagoides farina extract, Greer Laboratories, Lenoir, NC, USA)-induced atopic dermatitis model, both surfaces of the ear lobes were very gently stripped three times with Tegaderm. After stripping, $20 \mu \mathrm{l}$ of HDM $(10 \mathrm{mg} / \mathrm{ml})$ was painted on each ear. The application of HDM was repeated 3 times per week for 8 weeks. Two weeks after the first induction, blood was collected from the tail vein to measure serum IgE.

\section{Histology}

Ear tissues were fixed with $10 \%$ formalin and embedded in paraffin. Fixed tissues were cut into $5 \mu \mathrm{m}$ sections and deparaffinized. Sections were stained with hematoxylineosin (H\&E) or toluidine blue for light-microscopic examinations or mast cell infiltration assays, respectively. The epidermis and dermis thicknesses were measured using the iSolution DT36 software (Carl Zeiss, Oberkochen, Germany). The inflammation severity was scored as follows: ${ }^{26} 0$, none; 1 , minimal; 2, mild; 3 , moderate; and 4, marked. Terminal deoxynucleotidyl transferase dUTP nick end labeling (TUNEL) staining was performed using a commercial kit (Promega, Madison, WI, USA). For confocal microscopic analysis, sections were stained with antibodies against caspase-1 (14F468) or ASC (N-15) (both from Santa Cruz Biotechnology). Images were acquired with an LSM510 confocal laser scanning microscope (Carl Zeiss).

\section{Enzyme-linked immunosorbent assay (ELISA)}

The levels of IgE, IgG1, IgG2a, IL-1 $\beta$, TNF- $\alpha$, IFN- $\gamma$ IL4, IL-13, IL-17 (eBioscience, San Diego, CA, USA), histamine (Enzo Life Sciences), high-mobility group box 1 (HMGB1, Chondrex, Redmond, WA, USA), and lactate dehydrogenase (LDH) activity (Biovision, Milpitas, CA, USA) in mouse serum were measured using commercial ELISA kits.

\section{Cell surface staining and intracellular cytokine staining for flow cytometry}

Single-cell suspensions prepared from draining lymph nodes (dLNs) were stained with anti-CD4-PerCP/Cy5.5 
and anti-CD69-FITC antibodies (eBioscience) for $30 \mathrm{~min}$ on ice and washed with fluorescence-activated cell sorting buffer (2\% FBS in PBS). The cell surface expression of CD69 and percentage of IL-4-producing $\mathrm{CD}^{+}{ }^{+} \mathrm{T}$ cells were evaluated by flow cytometry on an Accuri flow cytometer (BD Biosciences, San Jose, CA, USA). All of the antibodies used were purchased from eBioscience.

\section{Statistical analysis}

Data are expressed as the means \pm SEMs. Significant differences between groups were determined using Student's unpaired $t$-test followed by Fisher's post-hoc analysis. A value of $p<0.05$ was accepted as an indication of statistical significance.

\section{Results}

Liquid chromatographic analysis shows increased $n-3$ PUFA content in fat-1 mice

We first quantified the major n-3 and n-6 PUFAs in skin tissues of fat-1 mice and their wild-type (WT) littermates. The n-3 PUFA (ALA, EPA, and DHA) content was significantly different between the genotypes, with higher levels in the serum and skin tissues of fat-1 mice (Table S1). Conversely, the n-6 PUFA (AA and LA) contents were lower in fat-1 mice compared with WT mice. Fat-1 mice had a skin tissue n-6/n-3 ratio of 5.6 compared to the ratio of 12.5 in WT mice.

\section{Fat-1 gene overexpression ameliorates the symptoms of} atopic dermatitis in mice

To validate the effects of endogenously synthesized n-3 PUFAs on atopic dermatitis, a DNCB-induced atopic dermatitis model was used (Fig. 1a). Following DNCB treatment, the ears of wild-type mice became red and swollen. On day 42, the ears of DNCB-treated wild-type mice showed severe erythema, erosion, and dryness (Fig. 1b). However, these atopic skin lesions were markedly attenuated in fat-1 mice. When ear thickness was measured over time and compared between genotypes, we found that thickness increased as atopic dermatitis developed. Consistent with the photographic images of the skin lesions, ear thickness was significantly reduced beginning on Day 7 (Fig. 1c). As IgE production, histamine release, and TSLP expression are markers for the development of atopic dermatitis, we also measured those levels in serum. As expected, fat-1 mice produced significantly less histamine, IgE, IgG1, IgG2a, and TSLP than wild-type mice at Day 43 (Fig. 1d, e). These data indicate

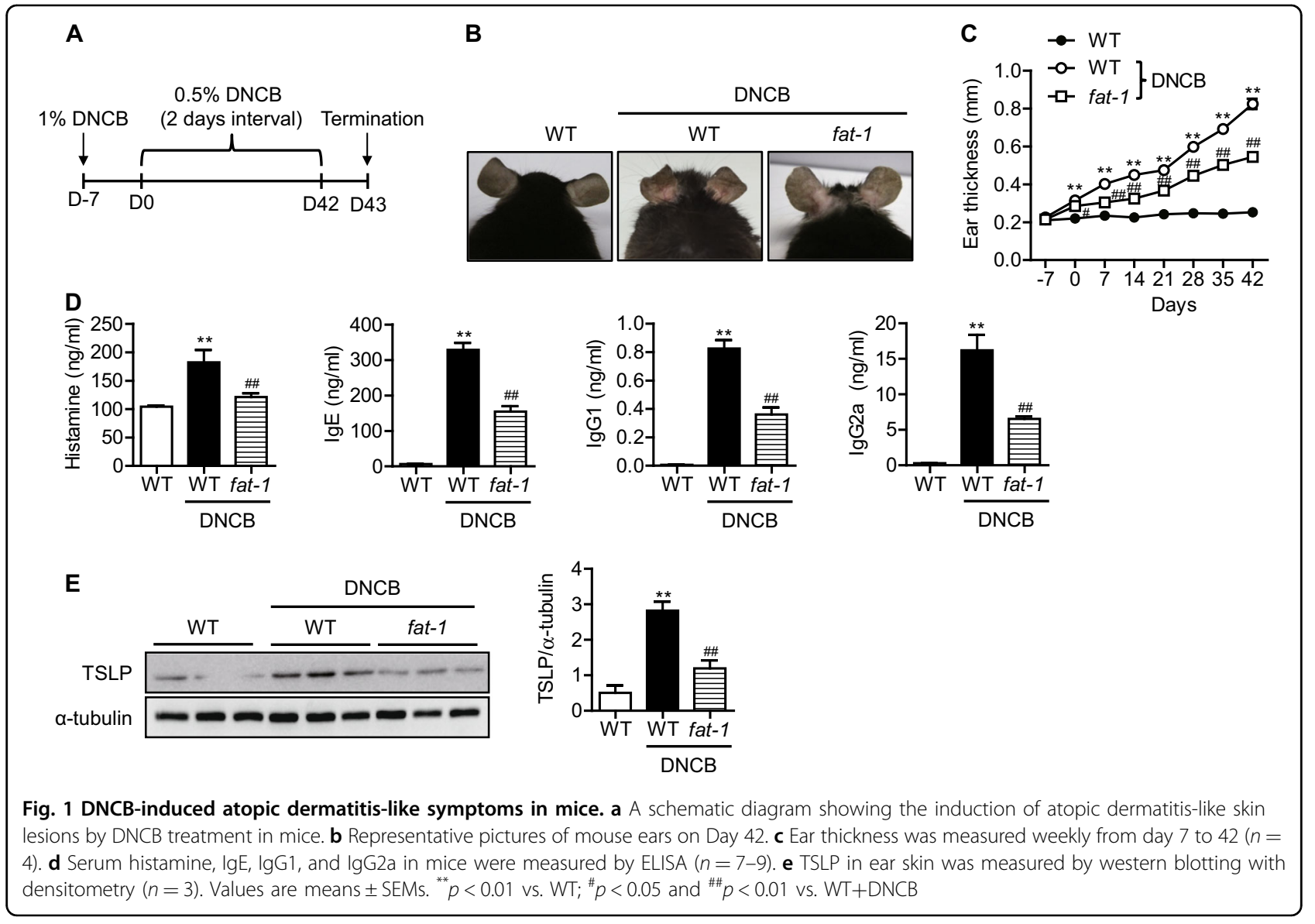


that endogenously synthesized n-3 PUFAs effectively ameliorate atopic dermatitis-like skin lesions in mice.

\section{Tissue inflammation and immune cell infiltration are diminished in fat-1 mice}

Improvements in the atopic symptoms of fat-1 mice were also confirmed by microscopic analysis of H\&Estained sections from ears removed at the end of the study. Wild-type mice under DNCB treatment exhibited the typical microscopic characteristics of atopic dermatitis, which include hyperkeratosis, parakeratosis, and acanthosis, along with infiltration of inflammatory cells (Fig. 2a, b). Toluidine blue staining of ear tissue sections revealed mast cell infiltration into the dermis of wild-type mice (Fig. 2a, b). However, in skin tissues from fat-1 mice, the epidermal and dermal tissues were significantly thinner and the infiltration of inflammatory cells into the dermis was significantly reduced. Accordingly, the inflammation score and mRNA levels of inflammationrelated genes were changed (Fig. 2c, d). These results suggest that reductions in inflammation and mast cell infiltration in fat-1 mice might attenuate the symptoms of atopic dermatitis. Since histamine release was markedly downregulated in fat-1 skin tissues (Fig. 1d), we hypothesized that endogenously synthesized n-3 PUFAs improved atopic dermatitis-like skin symptoms through downregulation of mast cell function. To test this possibility, we focused on the transcription factors required for mast cell activation. Gene expression analysis showed that Gata2, but not Gata1, was markedly increased by DNCB treatment in WT mice and significantly downregulated in fat-1 mice (Fig. 2e). The expression of the GATA2 target genes Cpa3 and c-Kit was altered accordingly.

\section{T cell activation and Th2 cytokine production are suppressed in draining lymph nodes of fat-1 mice}

To investigate whether skin inflammation increased the number of Th2 cells in the dLNs of the ear, submandibular lymph nodes were collected. We first observed that the lymph nodes in fat-1 mice were

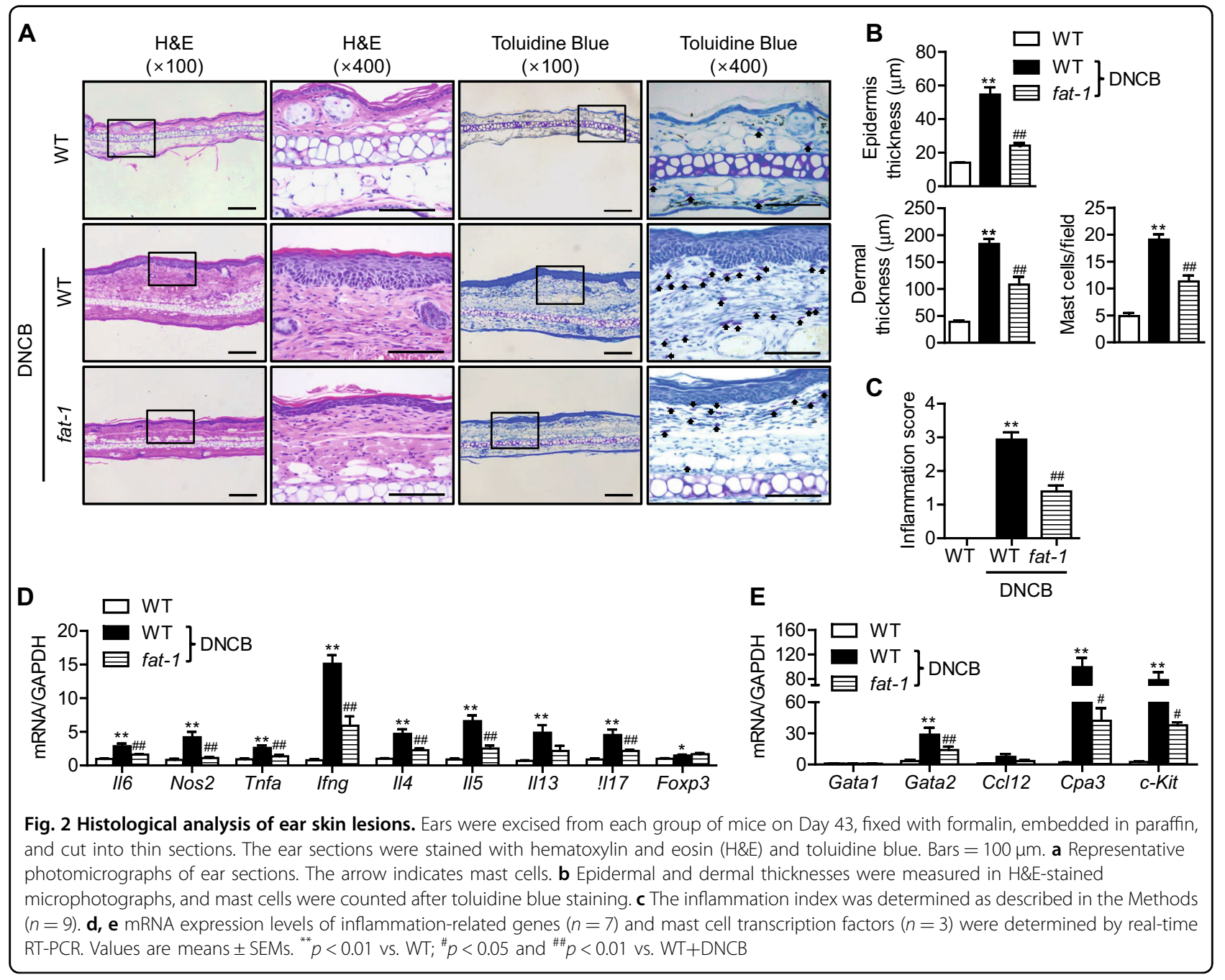



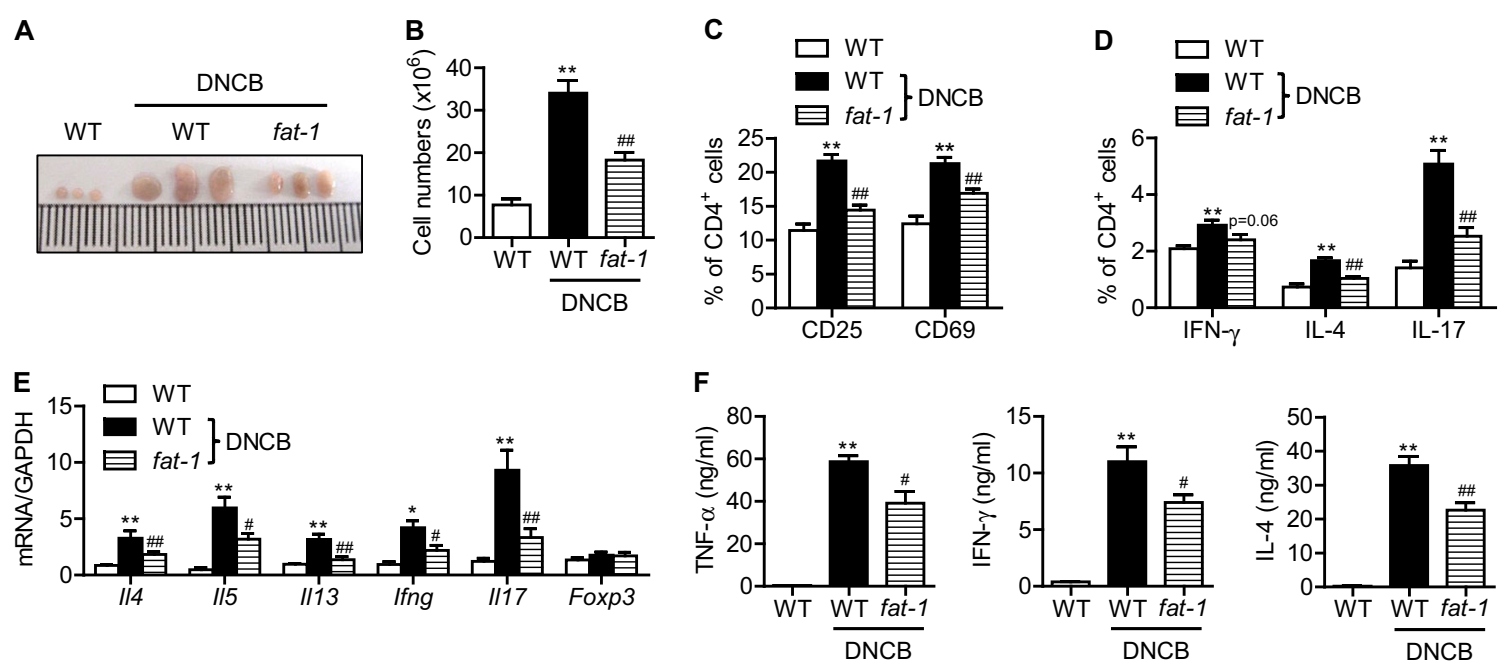

Fig. 3 T cell activation and differentiation in the draining lymph nodes. a At the end of the study, submandibular lymph nodes were collected, and photographs were taken. $\mathbf{b}$ Single-cell suspensions were prepared from dLNs, and total cells were counted $(n=9)$. c $C D 4^{+}$T cells were analyzed for CD25 and CD69 by flow cytometry $(n=9)$. $\mathbf{d ~ C D 4}{ }^{+} \mathrm{T}$ cells were stained for IL-4, IL-17, and IFN- $\gamma$ and analyzed by flow cytometry. The percentage of $\mathrm{CD}^{+}$cells expressing each cytokine is shown $(n=9)$. e dLNs were homogenized, and the mRNA levels of cytokines were analyzed by real-time RT-PCR $(n=7)$. f Serum cytokines were measured by ELISA $(n=7)$. Values are means \pm SEMs. ${ }^{* *} p<0.01$ vs. WT: $; p<0.05$ and ${ }^{\# \#} p<0.01$ vs. WT + DNCB

substantially smaller than those from wild-type mice (Fig. 3a). Lymph nodes were homogenized, and single cells were prepared and counted. Consistent with the lymph node size, the total cell numbers were significantly decreased in the lymph nodes of fat-1 mice (Fig. 3b). Next, single cells prepared from lymph nodes were analyzed for $\mathrm{T}$ cell activation. Flow-cytometric analysis showed that the percentages of $\mathrm{T}$ cells expressing $\mathrm{CD} 25^{+}$and $\mathrm{CD} 69^{+}$ were significantly lower in the dLNs of fat-1 mice (Fig. 3c), indicating that fat-1 expression suppressed DNCB-induced $\mathrm{CD}^{+} \mathrm{T}$ cell activation in dLNs. Moreover, $\mathrm{CD} 4^{+} \mathrm{T}$ cells from fat-1 mice showed lower percentages of Th1, Th2, and Th17 cells after DNCB treatment (Fig. 3d), but no difference in the percentage of Tregs (data not shown). Real-time RT-PCR analysis of cytokines in dLNs revealed an inhibition of $\mathrm{CD}_{4}^{+} \mathrm{T}$ cell differentiation into Th1, Th2, or Th17 in fat-1 mice (Fig. 3e). ELISA analysis in serum further revealed an inhibition of $\mathrm{CD} 4^{+} \mathrm{T}$ cell differentiation in fat-1 mice (Fig. 3f).

\section{n-3 PUFAs alleviate NLRP3 inflammasome-mediated pyroptosis by inhibiting NF-KB activation}

Since inflammasomes play important roles in skin inflammation, including atopic dermatitis ${ }^{27,28}$, we hypothesized that endogenously synthesized n-3 PUFAs would ameliorate the symptoms of atopic dermatitis by suppressing inflammasome activation. To test this hypothesis, we compared the protein and mRNA levels of NLRP3, ASC, and caspase-1, which are three key components of inflammasomes, in the skin of ears. NLRP3, procaspase-1, its proteolytic cleavage products $\mathrm{p} 20$ and $\mathrm{p} 10$, and ASC were markedly increased by DNCB treatment of WT mice (Fig. 4a, b). In agreement with the results of western blotting, immunofluorescence staining showed that p20 caspase-1 and ASC were elevated in DNCB-treated WT skin tissues (Fig. 4c). We further determined the protein levels of IL-1 $\beta$ and HMGB1, hallmarks of inflammasome activation. DNCB treatment increased the expression of IL-1 $\beta$ and HMGB1 in the skin tissues as well as their secretion into the systemic circulation (Fig. 4d, e). However, all of the aforementioned findings of inflammasome activation were remarkably suppressed in fat-1 mice.

Activation of inflammasomes culminates in a special type of cell death called pyroptosis, a proinflammatory programmed cell death. Cell death by pyroptosis is characterized by the loss of the plasma membrane integrity and eventual cell lysis ${ }^{29}$. We observed a significant decrease in TUNEL-positive cells in fat-1 mice compared with WT mice (Fig. S1A). LDH activity was also decreased in both the skin and serum of fat-1 mice, suggesting maintenance of membrane integrity (Figs. S1B and S1C).

Because NLRP3 expression is under transcriptional control of NF- $\kappa B$, we next evaluated changes in NF- $\kappa B$ under our experimental conditions. Acetylated p65 (Ac-p65) was markedly increased by DNCB, while no change was observed for total p65 (Fig. 5a). DNCB-mediated NF- $\kappa B$ activation was followed by an increase in the mRNA levels of two of the NF-kB-dependent proinflammatory molecules, Tnfa and $I l 1 b$ (Figs. $4 \mathrm{~b}$ and $5 \mathrm{~b}$ ). We further confirmed NF-kB activation by these cytokines in $\mathrm{HaCaT}$ cells 

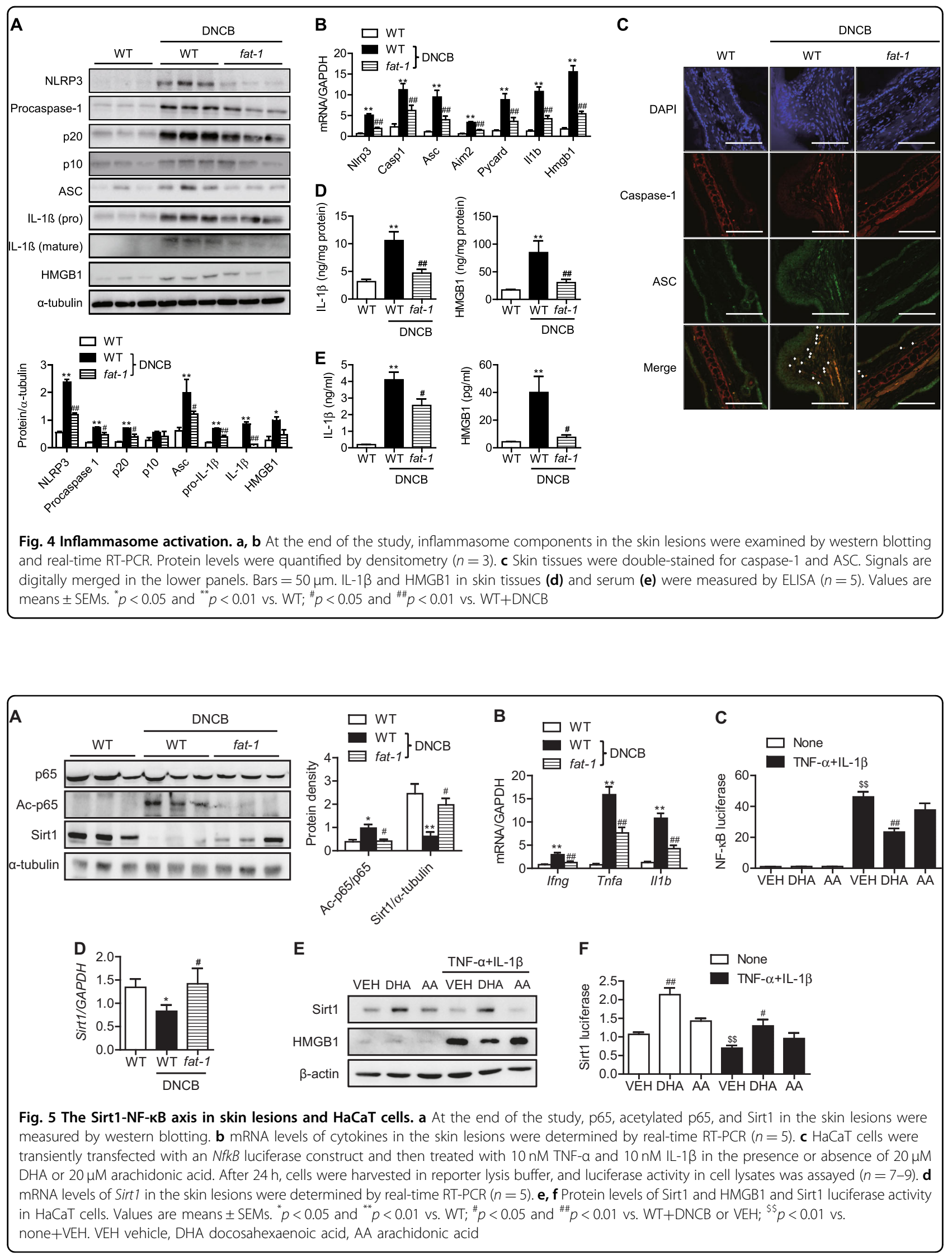
(Fig. 5c). Endogenously synthesized n-3 PUFAs in fat-1 mice and exogenous treatment with DHA in $\mathrm{HaCaT}$ cells significantly suppressed DNCB- and cytokine-induced NF$\kappa B$ activation, respectively (Figs. $5 \mathrm{a}$ and $5 \mathrm{c}$ ). However, arachidonic acid, an n-6 PUFA, had no effect on NF- $\mathrm{kB}$ activation.

We previously reported that fat-1 gene overexpression increased Sirt1 expression and decreased p65 acetylation $^{21}$. We therefore investigated whether Sirt1 expression was linked to the acetylation status of p65 in our experimental conditions. Our results revealed a significant reduction in Sirt1 expression and/or activity in DNCBtreated skin tissues and cytokine-treated $\mathrm{HaCaT}$ cells (Fig. 5d-f). Again, endogenous and exogenous n-3 PUFAs significantly upregulated the level of Sirt1 mRNA or protein.

\section{HDM-induced atopic dermatitis-like skin lesions are suppressed in fat-1 mice}

To explore the relevance of our findings to human disease, we reproduced our experiments using the HDM antigen, one of the most clinically important allergens for atopic dermatitis. HDM exposure induced increases in ear thickness; increases in serum HMGB1, IgE, and histamine; and histological abnormalities (Fig. 6a-e). Similar to the results obtained with the DNCB model, all of these parameters were significantly suppressed in fat-1 mice. The overall atopic symptoms and skin lesions observed in the HDM model were weaker than those in the DNCB model. Collectively, our experiments with HDM confirm our results with the DNCB model of atopic dermatitis.

\section{Discussion}

Several human clinical trials have addressed the beneficial effects of n-3 PUFAs on atopic dermatitis. Fish oil supplementation during pregnancy has resulted in improvements in the clinical severity of atopic outcomes for infants ${ }^{30,31}$. Similarly, infants supplemented with DHA-rich formula during the first year of life had a reduced incidence of atopic dermatitis during early childhood $^{32}$. Regulation of epidermal barrier function and modulation of the immune system have been suggested as underlying mechanisms ${ }^{33}$. Since DHA and EPA are not major constituents of the epidermis because of
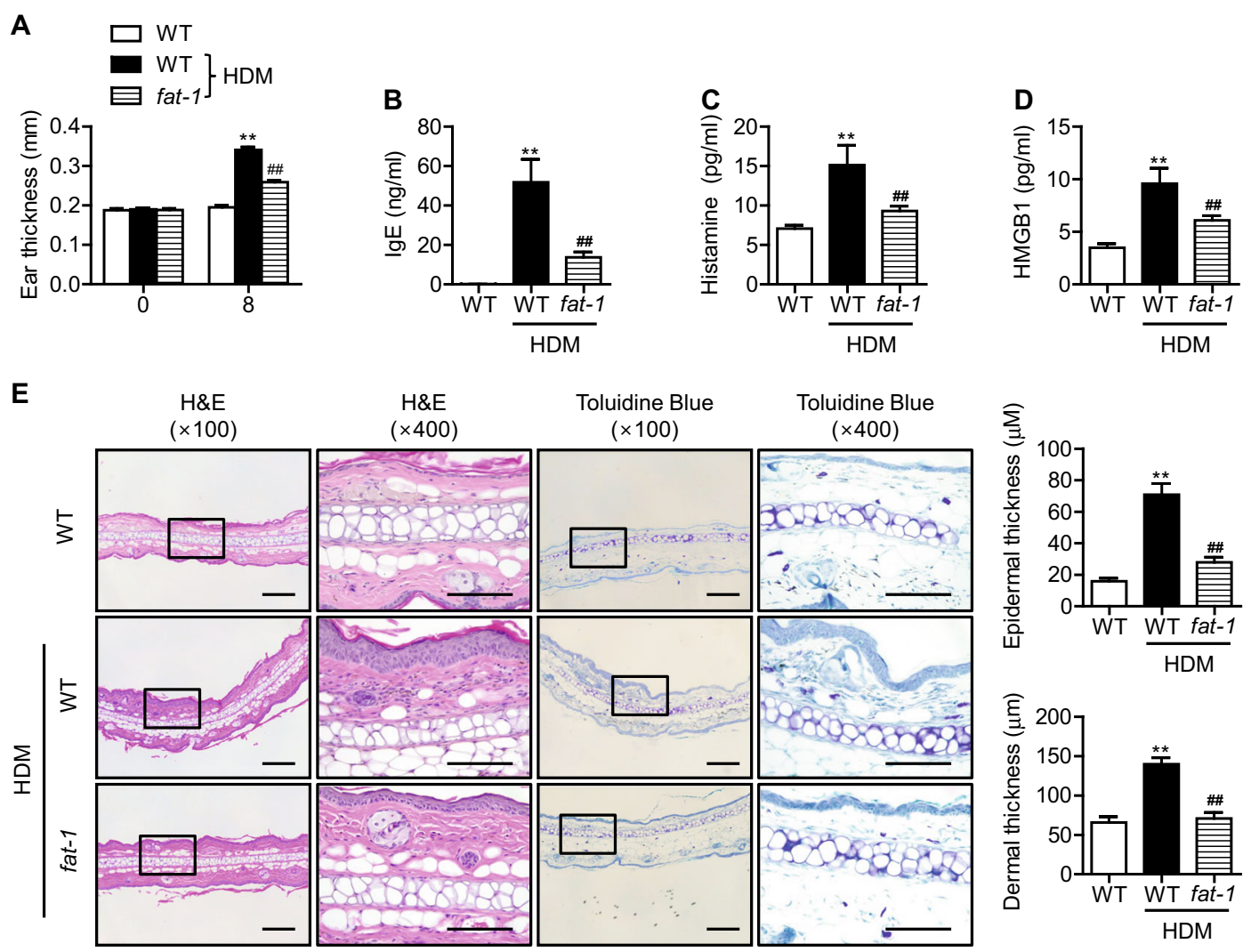

Fig. 6 Allergic inflammation in response to HDM. Mice were challenged with HDM as described in the Materials and Methods. a Ear thickness and (b-d) serum IgE, histamine, and HMGB1were analyzed $(n=6)$. e Paraffin-embedded skin sections were prepared and stained with H\&E and toluidine blue. Bars $=100 \mu \mathrm{m}$. Epidermal and dermal thicknesses were measured in H\&E-stained microphotographs $(n=6)$. Values are means \pm SEMs. ${ }^{* *} p<0.01$ vs. WT; ${ }^{\# \#} p<0.01$ vs. WT+HDM 
insufficient dietary consumption or increased cellular utilization, the restoration of skin permeability-barrier abnormality is not likely the main mechanism. Instead, substantial attention has been paid to the immunemodulatory function of $n-3$ PUFAs, especially $T$ cell regulation ${ }^{34,35}$. However, to our knowledge, a study on the inflammasome, a component of the innate immune system, has not been reported.

An Nlrp3 gene polymorphism has been associated with increased risk of atopic dermatitis ${ }^{11-13}$, and activation of inflammasomes triggers the allergic inflammatory response $^{36}$. Consequently, targeting the inhibition of NLRP3 inflammasomes is an attractive strategy for treating allergic diseases, including atopic dermatitis. Although there is growing evidence that nutrition is an effective modulator of the inflammasome, few attempts have been made to inhibit the NLRP3 inflammasome through diet. Interestingly, saturated fatty acids activate the NLRP3 inflammasome ${ }^{37,38}$, while unsaturated fatty acids prevent its activation ${ }^{39,40}$. Herein, we investigated whether endogenously synthesized n-3 PUFAs are effective in suppressing the NLRP3 inflammasome and mitigating the pathophysiological consequences of inflammation in mouse models of DNCB- and HDMinduced atopic dermatitis-like skin lesions. Our results showed that DNCB treatment resulted in activation of NLRP3 inflammasome signaling pathways, as evidenced by increases in NLRP3 and ASC expression, enhanced maturation of caspase- 1 and IL-1 $\beta$, and induction of pyroptosis. However, fat-1 gene overexpression increases Sirt1 expression, which allows for deacetylation of NF- $\mathrm{KB}$, resulting in the loss of its transcriptional activity. Given that NF- $\mathrm{KB}$ activation is required for the transcription of inflammasome components, including $N \operatorname{lrp} 3^{41}, \mathrm{n}-3$ PUFAs seem to inhibit the priming of the NLRP3 inflammasome based on its ability to suppress NF-kB.

Most of the mechanistic studies on atopic dermatitis point to an imbalance between Th1 and Th2 responses in favor of Th2 responses ${ }^{2,3}$. The characteristic Th2 responses observed in atopic dermatitis patients include abnormal IgE production, mast cell infiltration, peripheral eosinophilia, and induction of Th2 cells expressing IL-4 and IL-13. Interestingly, sequential biopsies from atopic dermatitis patients after exposure to aeroallergens show a biphasic immunologic response characterized by switching from a Th2 phenotype in the earlier phase to a Th1 phenotype in the later phase of the disease ${ }^{8}$. Concordantly, B cells switch their expression of immunoglobulin classes from IgE to IgG2a. Consistent with our previous report ${ }^{19}$, fat-1 gene overexpression decreased IL4, IL-5, IL-13, and IFN- $\gamma$ as well as IgE, IgG1, and IgG2a, suggesting that $\mathrm{n}-3$ PUFAs inhibit both Th2 and Th1 immune responses. In addition to the Th1/Th2 paradigm, the roles of Th17 and Treg cells in the pathogenesis of atopic dermatitis have also been highlighted ${ }^{42,43}$. IL-17 modulates innate immunity via keratinocytes, as IL-17 together with IFN- $\gamma$ amplifies a nonspecific cytotoxic cascade that results in a severe and sustained cutaneous inflammatory reaction ${ }^{44}$. To clarify this point, it was necessary to examine Th17 cell infiltration into skin lesions of fat-1 mice. In the present study, fat-1 gene overexpression successfully inhibited Th17 cell infiltration in the skin tissues and IL-17 production by draining lymph nodes, which was related to the decreased production of the inflammatory cytokines IFN- $\gamma$, TNF- $\alpha$, and IL-6. Consistent with our previous report ${ }^{19}$, the present study strongly suggests that fat-1 gene overexpression suppresses Th17 cell-mediated cutaneous inflammation in DNCB-treated mice. However, in contrast to the report by Han et $\mathrm{al}^{34}$, we did not observe an increase in Treg cells in fat-1 mice. In their study, DHA upregulated the function of M2 macrophages and promoted the generation of Treg cells. Since we also observed an increase in M2 macrophages in fat-1 mice ${ }^{21}$, differences in the $\mathrm{n}-3$ PUFAs (DHA in their study vs. n-3 PUFAs in our study) might have produced the different outcomes.

In summary, our data demonstrate that DNCB treatment activates the NLRP3 inflammasome pathway, which induces the release of inflammatory cytokines and amplifies the inflammatory response to cause atopic dermatitis symptoms. Modulation of this pathway with $n-3$ PUFAs has clinical relevance and can be a useful strategy to attenuate atopic dermatitis.

\section{Acknowledgements}

This work was supported by the Basic Science Research Program (grant numbers NRF-2017R1A2B2005730 and 2018R1D1A1B07046553) and by the Medical Research Center Program (grant number NRF-2017R1A5A2015061) through the National Research Foundation (NRF) funded by the Korean government (MSIP). We thank Sang-Hyun Kim (School of Medicine, Kyungpook National University, Korea) for critical reading of the manuscript.

Conflict of interest

The authors declare that they have no conflict of interest.

\section{Publisher's note}

Springer Nature remains neutral with regard to jurisdictional claims in published maps and institutional affiliations.

Supplementary information accompanies this paper at https://doi.org/ 10.1038/s12276-018-0104-3.

Received: 4 February 2018 Revised: 9 March 2018 Accepted: 19 March 2018.

Published online: 13 June 2018

\section{References}

1. Liang, Y., Chang, C. \& Lu, Q. The genetics and epigenetics of atopic dermatitisfilaggrin and other polymorphisms. Clin. Rev. Allergy Immunol. 51, 315-328 (2016).

2. Brandt, E. B. \& Sivaprasad, U. Th2 cytokines and atopic dermatitis. J. Clin. Cell Immunol. 2, 110 (2011). 
3. Kabashima, K. New concept of the pathogenesis of atopic dermatitis: interplay among the barrier, allergy, and pruritus as a trinity. J. Dermatol. Sci. 70, 3-11 (2013).

4. Weidinger, S. \& Novak, N. Atopic dermatitis. Lancet 387, 1109-1122 (2016).

5. Ito, T. et al. TSLP-activated dendritic cells induce an inflammatory Thelper type 2 cell response through OX40 ligand. J. Exp. Med 202, 1213-1223 (2005).

6. Soumelis, V. et al. Human epithelial cells trigger dendritic cell mediated allergic inflammation by producing TSLP. Nat. Immunol. 3, 673-680 (2002).

7. Tatsuno, K., Fujiyama, T., Yamaguchi, H., Waki, M. \& Tokura, Y. TSLP directly interacts with skin-homing Th2 cells highly expressing its receptor to enhance IL-4 production in atopic dermatitis. J. Invest Dermatol. 135, 3017-3024 (2015).

8. Grewe, M. et al. Analysis of the cytokine pattern expressed in situ in inhalant allergen patch test reactions of atopic dermatitis patients. J. Invest Dermatol. 105, 407-410 (1995)

9. Jiao, D. et al. NOD2 and TLR2 ligands trigger the activation of basophils and eosinophils by interacting with dermal fibroblasts in atopic dermatitis-like skin inflammation. Cell Mol. Immunol. 13, 535-550 (2016).

10. Arend, W. P., Palmer, G. \& Gabay, C. IL-1, IL-18, and IL-33 families of cytokines. Immunol. Rev. 223, 20-38 (2008).

11. Macaluso, F. et al. Polymorphisms in NACHT-LRR (NLR) genes in atopic dermatitis. Exp. Dermatol. 16, 692-698 (2007).

12. Zhang, Q., Fan, H. W., Zhang, J. Z., Wang, Y. M. \& Xing, H. J. NLRP3rs35829419 polymorphism is associated with increased susceptibility to multiple diseases in humans. Genet Mol. Res 14, 13968-13980 (2015).

13. Bivik, C. et al. Genetic variation in the inflammasome and atopic dermatitis susceptibility. J. Invest Dermatol. 133, 2486-2489 (2013).

14. Belloni, B. et al. Low-dose anti-lgE therapy in patients with atopic eczema with high serum IgE levels. J. Allergy Clin. Immunol. 120, 1223-1225 (2007).

15. He, A., Feldman, S. R. \& Fleischer, A. B. Trends in atopic dermatitis management: comparison of 1990-1997 to 2003-2012. J. Drugs Dermatol. 17, 135-140 (2018).

16. Chong, M. \& Fonacier, L. Treatment of eczema: corticosteroids and beyond Clin. Rev. Allergy Immunol. 51, 249-262 (2016).

17. Hageman, J. H. et al. The impact of dietary long-chain polyunsaturated fatty acids on respiratory illness in infants and children. Curr. Allergy Asthma Rep. 12 564-573 (2012).

18. Faber, J. et al. Supplementation with a fish oil-enriched, high-protein medical food leads to rapid incorporation of EPA into white blood cells and modulates immune responses within one week in healthy men and women. J. Nutr. 141, 964-970 (2011).

19. Jang, H. Y., Lim, K., Lee, S. M. \& Park, B. H. Effects of n-3 PUFA on the CD4 ${ }^{+}$type 2 helper T-cell-mediated immune responses in Fat-1 mice. Mol. Nutr. Food Res 58, 365-375 (2014).

20. Brown, A. L. et al. Omega-3 fatty acids ameliorate atherosclerosis by favorably altering monocyte subsets and limiting monocyte recruitment to aortic lesions. Arterioscler. Thromb. Vasc. Biol. 32, 2122-2130 (2012).

21. Song, M. Y. et al. Enhanced M2 macrophage polarization in high n-3 polyunsaturated fatty acid transgenic mice fed a high-fat diet. Mol. Nutr. Food Res 60, 2481-2492 (2016).

22. Teague, H., Rockett, B. D., Harris, M., Brown, D. A. \& Shaikh, S. R. Dendritic cell activation, phagocytosis and CD69 expression on cognate T cells are suppressed by n-3 long-chain polyunsaturated fatty acids. Immunology 139 386-394 (2013).

23. Kang, J. X., Wang, J., Wu, L. \& Kang, Z. B. Transgenic mice: fat-1 mice convert n6 to n-3 fatty acids. Nature 427, 504 (2004).

24. Weylandt, K. H. et al. Reduction of inflammation and chronic tissue damage by omega-3 fatty acids in fat- 1 transgenic mice with pancreatitis. Biochim Biophys. Acta 1782, 634-641 (2008).
25. Woo, S. J. et al. Endogenous conversion of n- 6 to n-3 polyunsaturated fatty acids attenuates $\mathrm{K} / \mathrm{BxN}$ serum-transfer arthritis in fat- 1 mice. J. Nutr. Biochem 26, 713-720 (2015).

26. Kurashima, Y. et al. The enzyme Cyp26b1 mediates inhibition of mast cell activation by fibroblasts to maintain skin-barrier homeostasis. Immunity $\mathbf{4 0}$, 530-541 (2014)

27. Dai, X. et al. Mite allergen is a danger signal for the skin via activation of inflammasome in keratinocytes. J. Allergy Clin. Immunol. 127, 806-14. e1-4 (2011).

28. Iversen, L. \& Johansen, C. Inflammasomes and inflammatory caspases in skin inflammation. Expert Rev. Mol. Diagn. 8, 697-705 (2008).

29. Miao, E. A., Rajan, J. V. SpringerAmpamp; Aderem, A. Caspase-1-induced pyroptotic cell death. Immunol. Rev. 243, 206-214 (2011).

30. Furuhjelm, C. et al. Allergic disease in infants up to 2 years of age in relation to plasma omega-3 fatty acids and maternal fish oil supplementation in pregnancy and lactation. Pediatr. Allergy Immunol. 22, 505-514 (2011).

31. Denburg, J. A. et al. Fish oil supplementation in pregnancy modifies neonatal progenitors at birth in infants at risk of atopy. Pediatr. Res 57, 276-281 (2005).

32. Birch, E. E. et al. The impact of early nutrition on incidence of allergic manifestations and common respiratory illnesses in children. J. Pediatr. 156 902-906 (2010). 906 e1.

33. McCusker, M. M. \& Grant-Kels, J. M. Healing fats of the skin: the structural and immunologic roles of the omega- 6 and omega-3 fatty acids. Clin. Dermatol. 28, 440-451 (2010).

34. Han, S. C. et al. Docosahexaenoic acid alleviates atopic dermatitis by generating Tregs and IL-10/TGF- $\beta$-modified macrophages via a TGF- $\beta$-dependent mechanism. J. Invest Dermatol. 135, 1556-1564 (2015).

35. Park, B. K. et al. Omega-3 fatty acids suppress Th2-associated cytokine gene expressions and GATA transcription factors in mast cells. J. Nutr. Biochem 24, 868-876 (2013).

36. Herberth, G. et al. Endogenous metabolites and inflammasome activity in early childhood and links to respiratory diseases. J. Allergy Clin. Immunol. 136, 495-497 (2015).

37. Wen, $H$. et al. Fatty acid-induced NLRP3-ASC inflammasome activation interferes with insulin signaling. Nat. Immunol. 12, 408-415 (2011).

38. Reynolds, C. M. et al. Dietary saturated fatty acids prime the NLRP3 inflammasome via TLR4 in dendritic cells-implications for diet-induced insulin resistance. Mol. Nutr. Food Res 56, 1212-1222 (2012).

39. Yan, Y. et al. Omega-3 fatty acids prevent inflammation and metabolic disorder through inhibition of NLRP3 inflammasome activation. Immunity $\mathbf{3 8}$, 1154-1163 (2013).

40. Finucane, O. M. et al. Monounsaturated fatty acid-enriched high-fat diets impede adipose NLRP3 inflammasome-mediated IL-1 $\beta$ secretion and insulin resistance despite obesity. Diabetes 64, 2116-2128 (2015).

41. Bauernfeind, F. G. et al. Cutting edge: NF-kB activating pattern recognition and cytokine receptors license NLRP3 inflammasome activation by regulating NLRP3 expression. J. Immunol. 183, 787-791 (2009).

42. Guttman-Yassky, E. \& Krueger, J. G. Atopic dermatitis and psoriasis: two different immune diseases or one spectrum? Curr. Opin. Immunol. 48, 68-73 (2017).

43. Di Cesare, A., Di Meglio, P. \& Nestle, F. O. A role for Th17 cells in the immunopathogenesis of atopic dermatitis? J. Invest Dermatol. 128, 2569-2571 (2008).

44. Pennino, D. et al. IL-17 amplifies human contact hypersensitivity by licensing hapten nonspecific Th1 cells to kill autologous keratinocytes. J. Immunol. 184, 4880-4888 (2010). 